\title{
Influence du temps et de l'hygrométrie sur le comportement du gypse
}

\section{AUVRAY \\ F. HOMAND \\ D. HOXHA}

Laboratoire Environnement Géomécanique et Ouvrages École nationale supérieure de géologie, Institut national polytechnique de Lorraine rue du Doyen

Marcel-Roubault, BP 40 54501 Vandœuvre-lèsNancy Cedex christophe.auvray@ensg.inplnancy.fi francoise.homand@ensg.inplnancy.fr dashnor.hoxha@ensg.implnancy.fr.

\section{DIDIER}

Institut national de l'environnement industriel et des risques Direction des risques du sol et du sous-sol Parc technologique Alata, BP 2

60550 Verneuil-en-Halatte christophe.didier@ineris.fr
La carrière souterraine de gypse située sur la commune de Grozon (Jura) présente de nombreuses instabilités dont l'ampleur est fonction de l'âge d'exploitation des quartiers. Dans les quartiers les plus âgés, les mínéraux constitutifs sont dissous, illustration du viéllissement du massif rocheux, Les propriétés physiques et mécaniques instantanées sont peu différentes entre les quartiers. Cepenciant, le comportement mécanique différé est influencé par l'hygrométrie: plus celle-ci est élevée, plus les vitesses de déformation augmentent. Ces vitesses de déformation sont également plus rapides pour les quartiers les plus anciens.

Mots-clés : carrière souterraine, gypse, comportement mécanique différé, vieillissement.

\section{Mechanical behavior of gypsum influenced by time and hygrometry}

The underground gypsum mine of Grozon shows instabilities that are function of the mining age. The constitutive minerals in samples from older mining areas show dissolution traces. Concerning the physical properties and instantaneous mechanical behaviour. few differences among gypsum of different exploited ages are to be noted. However, gypsum is more dissolved in the oldest areas. Laboratory tests show that creep mechanical behaviour of this rock is strongly influenced by the hygrometry. The strain rate is higher for the samples coming from the oldest areas.
}

Key words s mine, gypsum, creep behaviour, ageing. 


\section{Introduction}

L'effet du temps sur le comportement d'un ouvrage souterrain non revêtu comprend différents phénomènes responsables de la dégradation des propriétés des matériaux :

- la dégradation des minéraux constitutif's de la roche par action physico-chimique conduisant à une diminution des caractéristiques mécaniques, l'effet de vieillissement;

- le comportement mécanique différé sous chargement constant, c'est-à-dire le fluage ;

- les variations d'hygrométrie et donc des conditions de saturation et de succion du milieu rocheux, dans les zones où les conditions d'aérage varient, ces sollicitations cycliques peuverit induire un endommagement dû au couplage hydro-mécanique.

Dans le cas des milieux fracturés, à ces mécanismes de dégradation de la roche, s'ajoute également l'évolution de la fracturation qui devient alors un facteur très important. Dans cet article, nous nous limitons à l'étude du vieillissement et du fluage d'une roche particulière, le gypse.

La carrière souterraine de Grozon (Jura) a été choisie pour ses quartiers d'âge d'exploitation différent et l'existence, dans les secteurs les plus anciens, d'instabilités prononcées de différents types. Le gypse appartient à la série stratigraphique du Keuper supérieur. L'ensemble du site a actuellement une superficie d'environ 500 hectares (Fig. 1), la carrière est exploitée depuis la fin du XIXe siècle par la technique des chambres et piliers abandonnés sur un ou deux niveaux localement superposés. Le recouvrement de cette exploitation est compris entre 20 et 50 mètres. L'ensemble du site présente de nombreuses failles qui recoupent l'exploitation. Le rejet de ces failles est généralement plurimétrique.

La puissance des deux bancs de gypse exploités est de 2 mètres (Fig. 2) ; ils sont séparés par des intercalaires marneux et dolomitiques. Les piliers mesurent généralement 6 mètres par 6 mètres et 2,5 mètres de hauteur. Le taux de défruitement est compris entre 75 et $78 \%$. Cependant, ce schéma d'exploitation a varié à

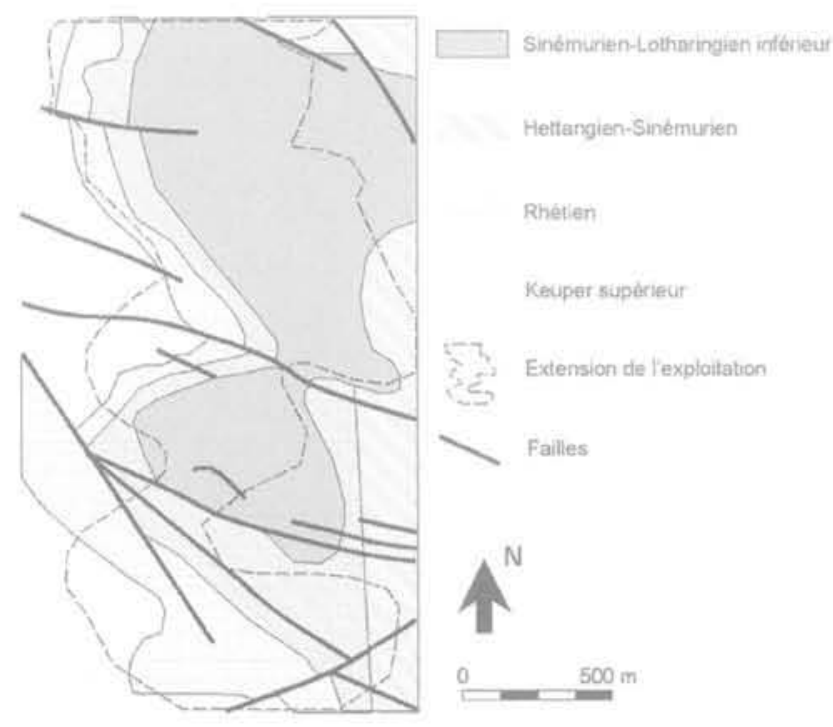

FIG.1 Carte géologique schématique du secteur de la carrière de Grozon. 
certains secteurs de travaux exploités en couche 3 , les piliers présentant en leur sommet une couche de marnes sensible au fluage.

Plusieurs mécanismes peuvent expliquer ces différents désordres:

- gonflement d'intercalaires marneux induisant des soufflages de mur;

- vieillissement des piliers conduisant à leur rupture et à des effondrements partiels,

Dans cet article, nous ne nous intéressons qu'à l'évolution des propriétés mécaniques des piliers de gypse en fonction du temps, c'est-à-dire au comportement différé ainsi qu'au vieillissement de la roche.

2.2

\section{Le gypse des piliers}

Le gypse est un sulfate de calcium dihydraté $\mathrm{CaSO}_{4}, 2 \mathrm{H}_{2} \mathrm{O}$; sa dureté est 2, il cristallise dans le système monoclinique (Ogniben, 1955 ; Jong et Bouman. 1959). Les paramètres de la maille élémentaire sont les suivants (Hejinen \& Hartman, 1991; Sipple, 1999): $a=5,6 \AA$ $\mathrm{b}=15,2 \AA ; \mathrm{c}=6,2 \AA$ et $\beta=114^{\circ}$. La représentation schématique de la maille élémentaire du système monoclinique est sur la figure 3 . Le cristal présente un clivage parfait $(010)$ oú les fractures obtenues sont lisses et deux autres clivages secondaires (100) et (011) où les fractures sont respectivement fibreuses et conchoïdales (Williams, 1988; Sipple, 1999).

Le gypse de la carrière de Grozon présente une pureté très variable, le pôle anhydrite peut en effet représenter $100 \%$ du matériau. Ce gypse est qualifié de microcristallin car les cristaux mesurent moins d'un millimètre de longueur.

Des différences entre des cristaux de gypse provenant de piliers de quartiers d'âge d'exploitation récent et de quartiers, d'âge d'exploitation ancien ont été mises en évidence. Des échantillons ont été étudiès au microscope électronique à balayage (MEB), ils proviennent de sondages horizontaux effectués dans des piliers. Dans le cas des quartiers récents, de nombreux cristaux sont automorphes et intacts. D'autres présentent des traces de dissolution sur les premiers cinquante centimètres vers le coeur du pilier (Fig. 4). Les cristaux pro-

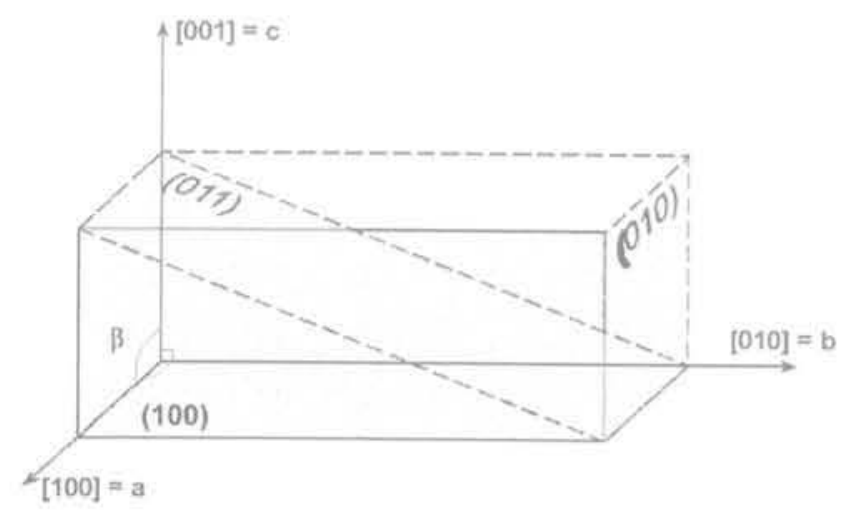

FG. 3 Représentation schématique de la maille élémentaire du réseau cristallín monoclinique du gypse (les 3 faces référencées sont les plans des 3 principaux clivages). venant d'échantillons prélevés dans les quartiers d'âge d'exploitation ancien présentent des dissolutions qui sont quantitativement plus importantes et observables jusqu'à plus d'un mètre de profondeur. Les cristaux sont tous xénomorphes du fait de la dissolution marquée en peau de pilier. L'intensité de la dissolution décrôt du parement vers le cœur du pilier, ce qui suggère un mécanisme dépendant du temps et donc un vieillissement de la roche. Des dissolutions avec ou sans néoformation de minéraux, ont déjà été décrites dans diverses roches (Farran et Thénoz, 1965; Auger, 1991; Furlan et Girardet, 1991; Chêne et al., 1999; Gupta et Rao, 2000; Grigc et al., 2002) avec des mécanismes qui dépendent de la nature minéralogique. Dans le cas du gypse, il ne peut s'agir que de dissolution.

\section{3}

\section{Caractérisation physique et mécanique instantanée}

Les caractéristiques physiques et mécaniques instantanées de ce gypse sont synthétisées dans le tableau I. Ces valeurs sont des moyennes calculées pour chaque âge d'exploitation, sur une vingtaine d'échantillons secs pour les paramètres physiques. La résistance moyenne en compression uniaxiale et en traction indirecte sur des échantillons secs a été déterminée à partir d'une dizaine d'essais.

La dispersion des résultats peut être importante. Elle a principalement une origine structurale car les échantillons présentent des fissures qui sont plus ou moins cicatrisées. La pureté de la roche peut également avoir un rôle, même si tous les échantillons sont constitués de 90 à $100 \%$ de gypse, l'autre constituant étant de l'anhydrite, qui modifie les caractéristiques physico-mécaniques de l'échantillon. Cette dispersion peut masquer en partie les variations des caractéristiques physiques et mécaniques instantanées qui sont fonction de l'âge de l'exploitation. Néanmoins, une diminution de $12 \%$ de la résistance en compression uniaxiale a été mesurée entre les échantillons secs provenant d'un quartier récent et ancien.

Quel que soit l'âge d'exploitation, le comportement mécaniqué instantané des échantillons secs de ce matériau reste élastoplastique avec une rupture qui devient ductile avec l'augmentation de la pression de confinement (Figs 5a et 5b). Cependant les déformations plastiques avant le pic sont assez faibles et le seuil de la dilatance est très proche du pic.

Les résistances au pic s'alignent raisonnablement bien sur une droite dans le plan p-q, un critère de type Mohr-Coulomb peut donc être ajusté (Fig. 6). Le calcul des paramètres de ce critère avec des échantillons secs montre que l'angle de frottement augmente de $43^{\circ}$ pour les échantillons de l'exploitation récente à $49^{\circ}$ pour les échantillons du quartier 1920. A l'inverse, la cohésion diminue de $9 \mathrm{MPa}$ à $7 \mathrm{MPa}$

La résistance en compression uniaxiale d'échantillons partiellement saturés en eau a été déterminée. Les échantillons ont été placés dans une atmosphère avec une hygrométrie constante. Les conditions d'hygrométrie choisies sont les suivantes: $30 \%, 60 \%$ et $90 \%$ à une température de $20^{\circ} \mathrm{C}$. La solution saline saturée provoquant une hygrométrie de $30 \%$ est composée de cristaux de sel de chlorure de calcium hexahydraté $\left(\mathrm{CaCl}_{2}, 6 \mathrm{H}_{2} \mathrm{O}\right)$. Avec ce sel, I'hygrométrie attendue est normalement de $36,5 \%$. L'hygrométrie de 


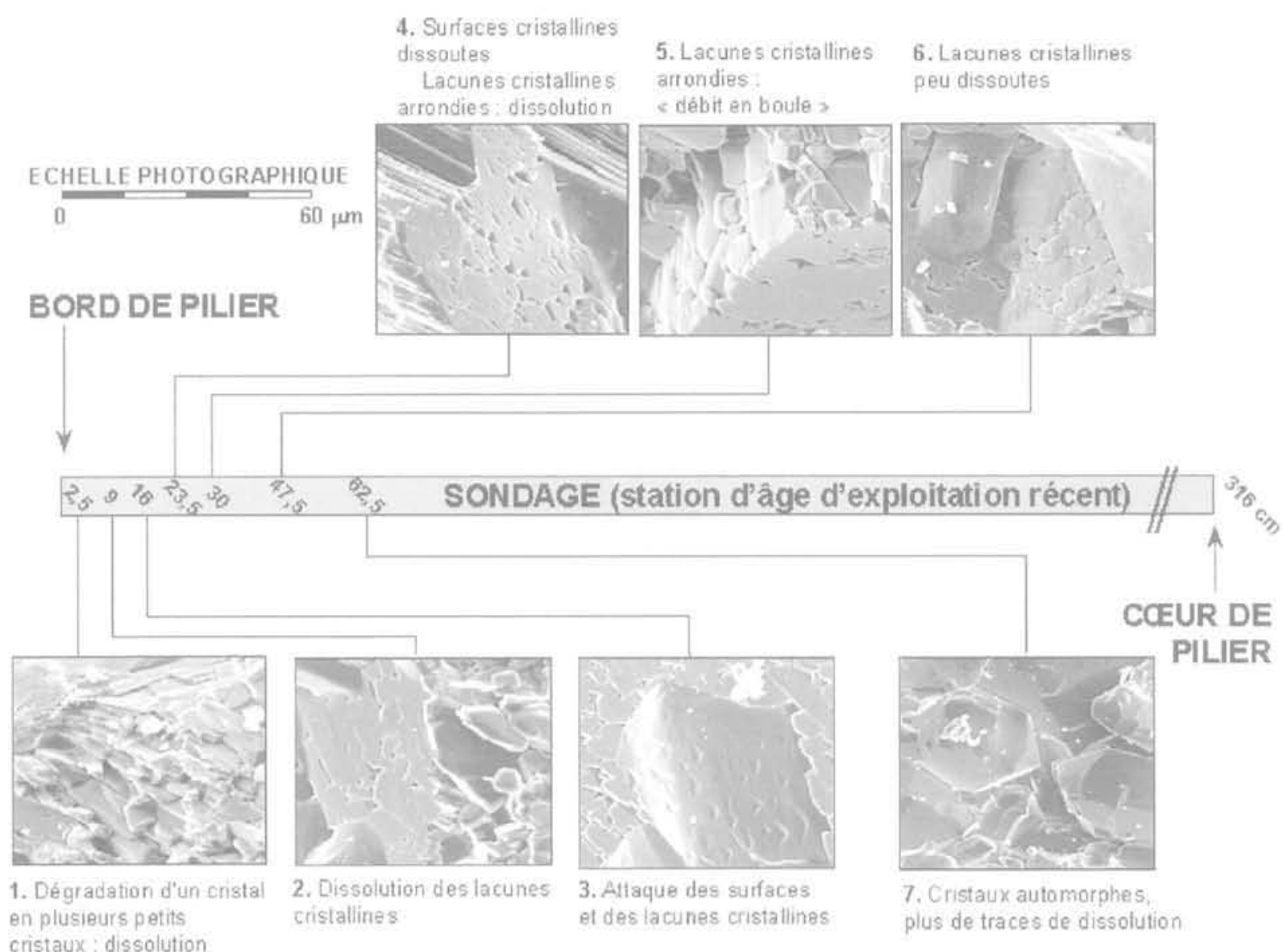

FG. 4 Évolution des traces de dissolution dans un pilier (quartier d'âge d'exploitation récent).

$60 \%$ est obtenue avec une solution saline saturée avec le sel de nitrite de sodium ( $\mathrm{NaNO}_{2}$ ). Ce sel donne normalement une hygrométrie de $66 \%$. Concernant la solution saline de $90 \%$, le sel est du sulfate de cuivre (II) pentahydraté ( $\left.\mathrm{CuSO}_{4}, 5 \mathrm{H}_{2} \mathrm{O}\right)$. Avec ce sel, l'hygrométrie attendue est de $98 \%$. L'essai de compression uniaxiale est réalisé lorsque la masse de l'échantillon ne varie plus. La résistance moyenne en compression uniaxiale des échantillons partiellement saturés a été calculée à partir de deux ou trois essais pour chacune des hygrométries et pour chacun des âges. Malgré la dispersion des résultats, nous constatons une tendance à la diminution de la résistance avec l'augmentation de I'hygrométrie (Tableau I). La diminution de résistance en compression simple entre des échantillons partiellement saturés provenant de quartiers d'âge d'exploitation différent ne dépasse pas $25 \%$ pour le cas le plus défavorable (quartier ancien, hygrométrie = $90 \%$ ).

\section{4}

\section{Comportement mécanique différé}

Le comportement différé du gypse a fait l'objet d'études antérieures (Griggs, 1939, 1940; Griggs et Handin, 1960; Morlier, 1964). Cependant, tous ces essais ont été réalisés dans des conditions dites standard, c'est-à-dire sans mesure des déformations différées transversales et sans contrôle de l'hygrométrie.

\section{1}

\section{L'équipement au laboratoire}

L'équipement nécessaire pour ce type d'essai est un bâti de fluage composé de trois principaux éléments l'embase, le corps et la tête. Les volumes du récipient et du corps de la cellule ont été choisis en fonction de la norme NF EN ISO 483 et par rapport aux dimensions des éprouvettes qui font $38 \mathrm{~mm}$ de diamètre et un élancement de 2.

L'atmosphère autour de l'éprouvette a une hygrométrie contrôlée et constante pendant toute la durée de l'essai. Pour générer cette hygrométrie, un récipient dans lequel se trouve une solution saline saturée vient se positionner sous l'embase du bâti. Tout le dispositif est placé dans une salle climatisée afin de pouvoir maintenir la température constante et la plus proche possible de $20^{\circ} \mathrm{C}$. La norme NF X 15-119 a servi de support technique pour les mesures d'hygrométrie en fonction de la température et les précautions d'emploi des solutions salines saturées.

Sur les éprouvettes sont collées des jauges d'extensométrie pour mesurer les déformations axiales et les déformations latérales. Il est cependant particulièrement difficile d'assurer le bon fonctionnement des jauges sous forte humidité relative. Une technique a donc été mise au point sans que le taux de réussite soit complet. Une sous-couche de résine époxy est appliquée sur la zone de collage des jauges. Cette résine 
TABLEAUI Caractéristiques physiques et mécaniques instantanées en fonction de l'âge d'exploitation du quartier. Anglais

\begin{tabular}{|c|c|c|c|c|c|c|}
\hline & \multicolumn{2}{|c|}{ Quartier 2000} & \multicolumn{2}{|c|}{ Quantier 1970} & \multicolumn{2}{|c|}{ Quartier 1920} \\
\hline & moyenne & écart-type & moyenne & ecart-type & moyenne & écart-type \\
\hline $\begin{array}{l}\text { Porosité totale : } n(\%) \\
\text { (12 échantillons par quartier) }\end{array}$ & 2,5 & 1,0 & 1.0 & 0,4 & 1.0 & 1,3 \\
\hline $\begin{array}{l}\text { Masse volumique des grains: } p_{\mathrm{v}}\left(\mathrm{g} / \mathrm{cm}^{3}\right) \\
\text { (12 échantillons par quartier) }\end{array}$ & 2,33 & - & 2,33 & - & 2,33 & - \\
\hline $\begin{array}{l}\text { Vitesse des ondes ultrasonores } P: V_{D}(\mathrm{~m} / \mathrm{s}) \\
\text { (12 échantillons par quartier) }\end{array}$ & 4850 & 180 & 4950 & 35 & 5000 & 100 \\
\hline $\begin{array}{l}\text { Vitesse des ondes ultrasonores } S: V_{,}(\mathrm{m} / \mathrm{s}) \\
\text { (12 échantillons par quartier) }\end{array}$ & 2400 & 75 & 2500 & 25 & 2500 & 30 \\
\hline $\begin{array}{l}\text { Essai de traction indirecte: résistance } \mathrm{R}_{\mathrm{q}}(\mathrm{MPa}) \\
\text { (12 échantillons par quartier) }\end{array}$ & 5,0 & 1,9 & 5 & 1,1 & 3,3 & 2,0 \\
\hline $\begin{array}{l}\text { Essai de compression simple: } \\
4 \text { échantillons secs par quartier résistance: } \mathrm{R}_{c}(\mathrm{MPa}) \\
\text { module d'Young: } \mathrm{E} \text { (GPa) }\end{array}$ & $\begin{array}{c}42 \\
31,3\end{array}$ & $\begin{array}{l}1,7 \\
5,9\end{array}$ & $\begin{array}{c}42 \\
34,8\end{array}$ & $\begin{array}{l}0,8 \\
3,2\end{array}$ & $\begin{array}{l}37,5 \\
34,4\end{array}$ & $\begin{array}{l}1,9 \\
1,2\end{array}$ \\
\hline $\begin{array}{l}\text { Essai de compression simple: } \\
4 \text { échantillons partiellement saturés } \\
\text { (Hr }=30 \% \text { ) par quartier } \\
\text { résistance: } R_{c} \text { (MPa) } \\
\text { module d'Young: } E \text { (GPa) }\end{array}$ & $\begin{array}{c}41 \\
32,5\end{array}$ & $\begin{array}{l}0,8 \\
2,5\end{array}$ & $\begin{array}{c}42 \\
48,1\end{array}$ & $\begin{array}{l}0,9 \\
5,2\end{array}$ & $\begin{array}{c}38 \\
35,1\end{array}$ & $\begin{array}{l}1,5 \\
3,8\end{array}$ \\
\hline $\begin{array}{l}\text { Essai de compression simple: } \\
4 \text { échantillons partiellement saturés } \\
\text { (Hr }=60 \% \text { ) par quartier } \\
\text { résistance: } \mathrm{R}_{c} \text { (MPa) } \\
\text { module d'Young: } \mathrm{E} \text { (GPa) }\end{array}$ & $\begin{array}{l}43,5 \\
30,2 \\
\end{array}$ & $\begin{array}{l}1,6 \\
4,3\end{array}$ & - & - & $\begin{array}{c}35 \\
32,1\end{array}$ & $\begin{array}{c}2 \\
2,9\end{array}$ \\
\hline $\begin{array}{l}\text { Essai de compression simple : } 4 \text { échantillons } \\
\text { partiellement saturés }(\mathrm{Hr}=90 \% \text { ) par quartier } \\
\text { résistance: } \mathrm{R}_{c}(\mathrm{MPa}) \\
\text { module d'Young: } \mathrm{E}(\mathrm{GPa})\end{array}$ & $\begin{array}{l}42,5 \\
35,1\end{array}$ & $\begin{array}{l}1,2 \\
2,4\end{array}$ & - & - & $\begin{array}{l}32,2 \\
34,9\end{array}$ & $\begin{array}{l}2,5 \\
1,9\end{array}$ \\
\hline
\end{tabular}

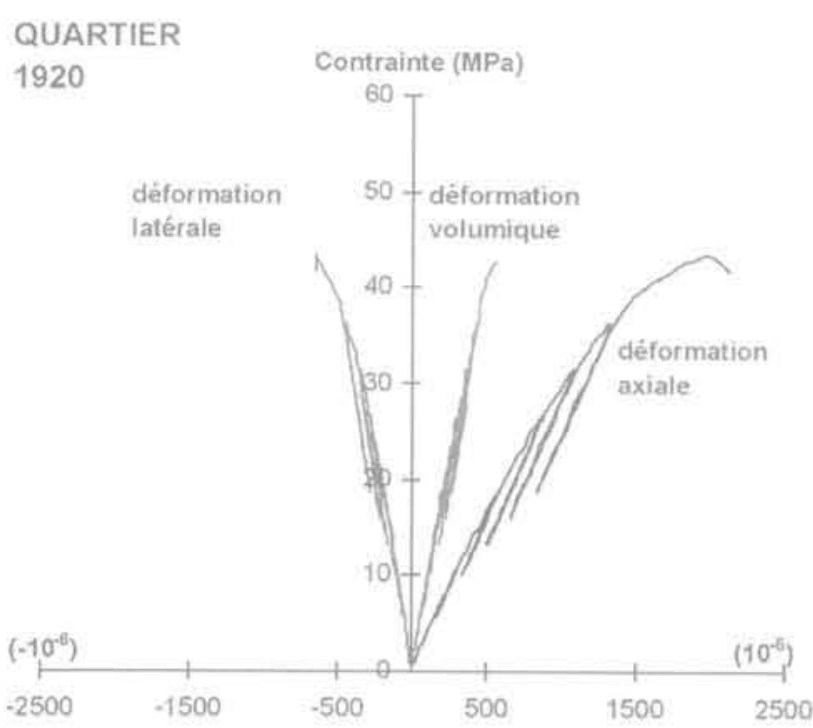

FIG.5A Exemple de courbes contraintedéformation d'un essai de compression uniaxiale (quartier d'âge d'exploitation 1920).

pénètre superficiellement dans le réseau poreux du matériau et sa surface est parfaitement lisse. Sans cette sous-couche, la jauge peut se décoller. Par la suite, un anneau de résine époxy de $1 \mathrm{~mm}$ d'épaisseur englobe les jauges et les soudures des fils pour les protéger

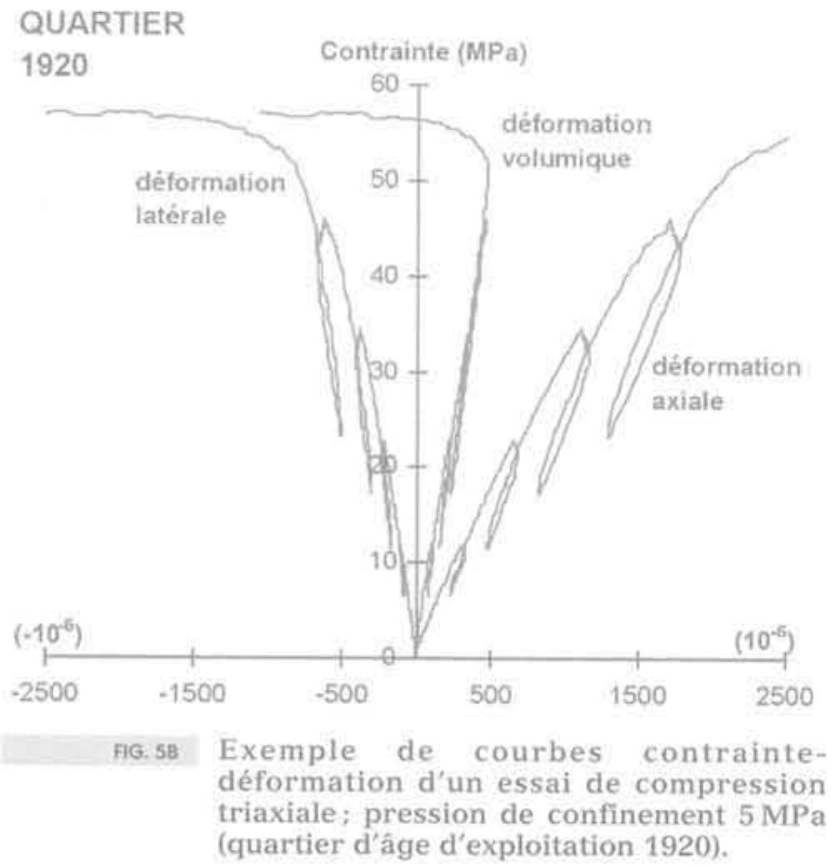

pendant la durée de l'essai de fluage qui est de plusieurs semaines. Pour minimiser l'influence de la rigidité de l'anneau de résine, quatre coupures verticales diamétralement opposées deux à deux ont été effectuées. 


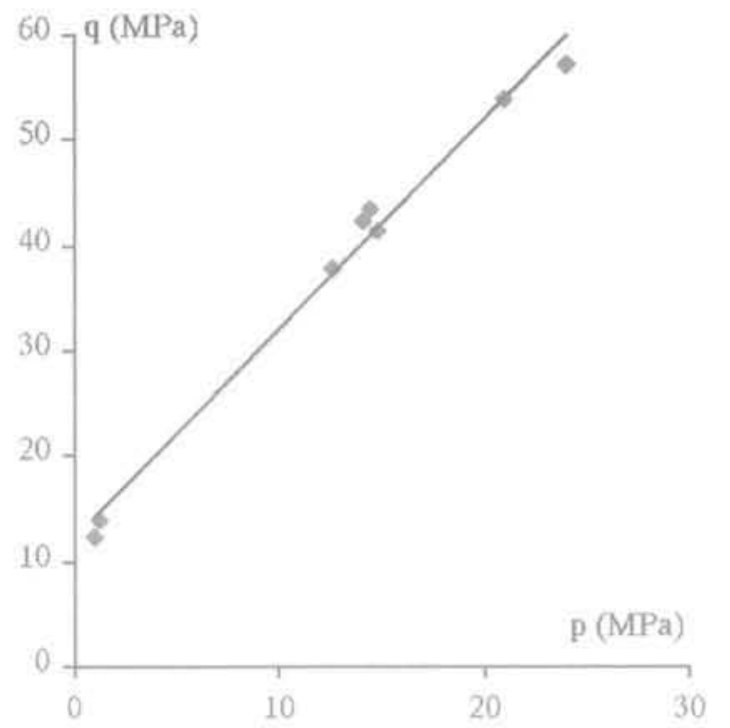

FIG.6 Exemple d'une reprèsentation du critère de rupture de Mohr-Coulomb (quartier d'âge d'exploitation 1920).

Le comportement mécanique différé est étudié pour une même contrainte axiale totale de $10 \mathrm{MPa}$ sous différentes hygrométries et à température constante. Les essais ont été réalisés à des hygrométries imposées de: $-30 \%$ correspondant à un état quasi sec:

- $90 \%$ correspondant à un état dans les quartiers récents en été;

- $60 \%$ pour avoir une valeur intermédiaire.

La contrainte n'a été appliquée qu'à partir d'une dizaine de jours après la mise des échantillons dans l'atmosphère à hygrométrie contrôlée. Le niveau de contrainte choisi pour ces essais correspond à environ
$25 \%$ de la résistance en compression uniaxiale des échantillons secs et il ne dépasse pas $30 \%$ de la résistance en compression simple pour le cas le plus défavorable (quartier ancien, $\mathrm{Hr}=90 \%$ ).

Neuf essais de fluage ont été réalisés: un âge d'exploitation pour chaque hygrométrie. Les éprouvettes utilisées pour les essais de fluage ont été prélevées entre 20 et $40 \mathrm{~cm}$ à partir du bord des piliers. Deux essais, avec des échantillons provenant du quartier en cours d'exploitation, ont été modifiés. Les échantillons ont subi un changement d'hygrométrie sans relâchement de la contrainte: pour le premier échantillon, une hygrométrie de $30 \%$ pendant 200 jours puis $90 \%$ pendant 15 jours et pour le second échantillon, une hygrométrie de $60 \%$ pendant 120 jours puis $90 \%$ pendant 15 jours.

\section{2}

\section{Résultats des essais de fluage à hygrométrie contrôlée}

Les résultats des différents essais de fluage à hygrométrie contrôlée sont présentés sur les figures 7 à 9. Pour les essais avec une hygrométrie de $90 \%$, une phase de fluage s'apparentant au fluage primaire dure environ 15 jours. A partir du $45^{\circ}$ jour environ, la phase tertiaire commence. Les déformations volumiques sont initialement contractantes pour devenir dilatantes. La manifestation de la dilatance volumique est fonction de l'âge (Tableau II). Le seuil de dilatance est d'autant plus vite atteint que l'âge d'exploitation de l'échantillon est ancien.

La durée de la phase du fluage primaire est plus longue pour une faible hygrométrie : elle est de 60 jours et de 80 jours environ pour les essais avec une hygrométrie de $60 \%$ et $30 \%$. Pour ces hygrométries, quand les essais ont été arrêtés, un fluage secondaire était observé depuis plusieurs semaines. Les déformations
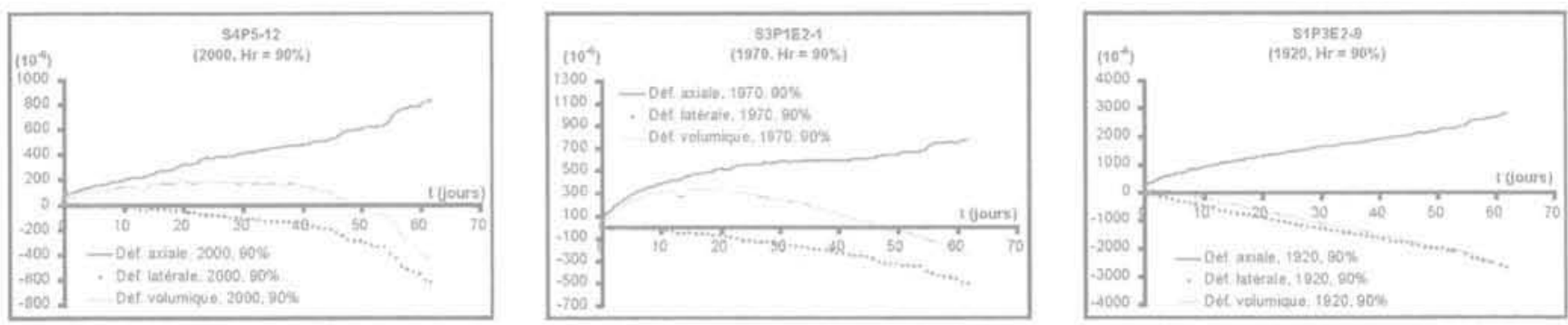

Fig. 7 Courbes déformation-temps de trois échantillons d'âge d'exploitation différent sous humidité relative de $90 \%$. Anglais
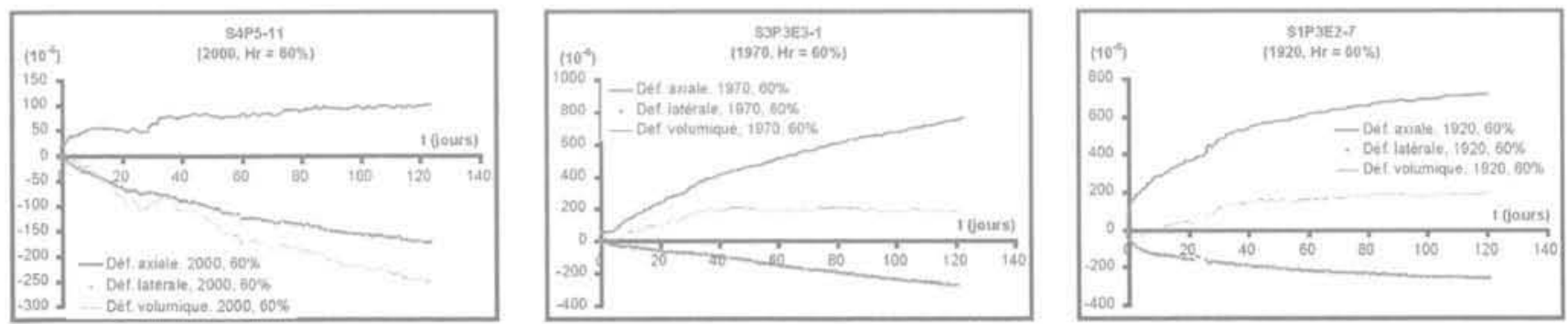

FG. 8 Courbes déformation-temps de trois échantillons d'âge d'exploitation différent sous humidité relative de $60 \%$. 

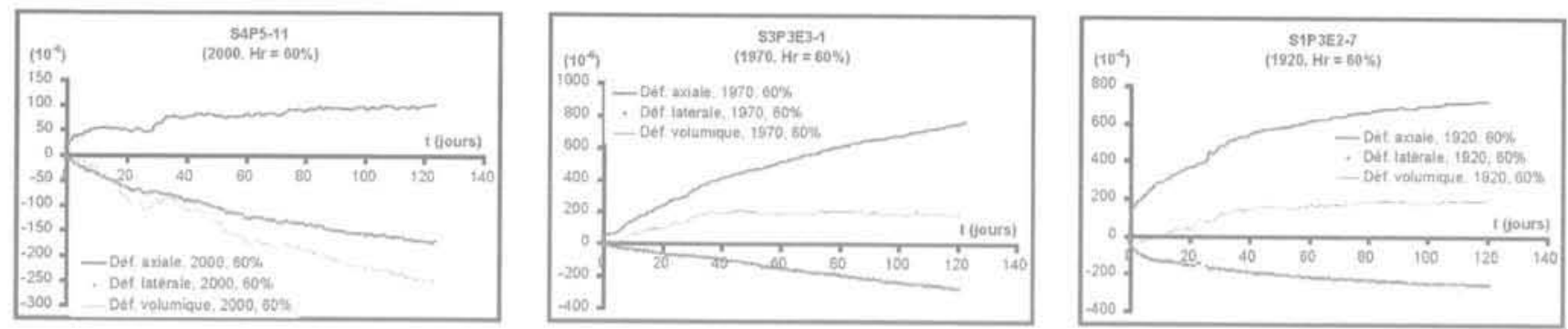

FiG.9 Courbes déformation-temps de trois échantillons d'âge d'exploitation différent sous humidité relative de $30 \%$.

TAateAuII Seuil de dilatance pour les 3 essais de fluage avec une hygrométrie de $90 \%$.

\begin{tabular}{l|c|c|c}
\hline Seuil de dilatance & Quartier 2000 & Quartier 1970 & Quartier 1920 \\
\hline $25^{\mathrm{e}}$ jour & $15^{\mathrm{r}}$ jour & $5^{\mathrm{e}}$ jour \\
\hline
\end{tabular}

volumiques des échantillons avec une hygrométrie de $60 \%$ sont initialement contractantes mais, au bout d'environ 6 semaines, la vitesse des déformations volumiques est constante, à l'exception de l'échantillon S4P5-11 pour lequel les déformations volumiques sont dilatantes dès le début du fluage (Fig. 8). Pour les échantillons avec une hygrométrie de $30 \%$, les déformations volumiques restent contractantes pendant toute la durée de l'essai.

Pour une hygrométrie constante, les déformations axiales et latérales au moment du passage fluage primaire-fluage secondaire sont fonction de l'âge d'exploitation de l'échantillon. Ces déformations sont d'autant plus élevées que le quartier de provenance a été exploité à une période ancienne. Pour un âge constant, les déformations axiales et latérales au moment du passage fluage primaire-fluage secondaire sont fonction de l'hygrométrie. A ce passage, les déformations sont plus importantes pour les fortes hygrométries.

Les vitesses des déformations axiales et latérales pendant le fluage secondaire ont été calculées à partir de la partie sensiblement linéaire des courbes de fluage (Tableau III). Pour une hygrométrie donnée, les vitesses sont fonction de l'âge d'exploitation de la zone de prélèvement: plus l'âge d'exploitation de la zone des prélèvements est ancien, plus la vitesse est élevée. Pour un àge donné, les vitesses sont fonction de l'hygrométrie: plus l'hygrométrie est importante, plus la vitesse est élevée.

La présence des déformations volumiques dilatantes aussi bien sur les courbes des essais instantanés que celles du fluage indiquent probablement un endommagement de la roche par une propagation cri- tique et/ou subcritique des físsures, assistée par des mécanismes chimiques et accélérée par l'eau. Afin de mieux comprendre et de confirmer ou infirmer ces hypothèses, des observations au microscope électronique à balayage (MEB) et des essais sur des éprouvettes ayant subi un fluage ont été réalisés.

\section{5}

\section{Paramètres physiques, observations microscopiques et essais mécaniques post fluage}

\author{
5.1
}

\section{Paramètres physiques avant et après essais de fluage}

Les vitesses des ondes ultrasonores de compression $\mathrm{P}\left(\mathrm{V}_{p}\right)$ et de cisaillement $\mathrm{S}\left(\mathrm{V}_{\mathrm{f}}\right)$ ont été mesurées sur des éprouvettes séchées à $50^{\circ} \mathrm{C}$ avant et après les essais de fluage. Ces mesures ont permis de calculer le module d'Young $\left(E_{\text {dun }}\right.$ ) et le coefficient de Poisson dynamiques $\left(v_{\text {dyn }}\right)$ (Tableau IV).

En règle gẻnérale, nous constatons une tendance à la diminution des vitesses des ondes ultrasonores et des modules d'Young dynamiques après le fluage. L'amplitude de cette diminution, tout en restant faible, est fonction de l'hygrométrie et de l'âge de l'exploitation.

La diminution du module d'Young dynamique est d'autant plus importante que l'hygrométrie est élevée et que la période d'exploitation de la station de prélévement est ancienne. D'une manière générale, la diminution du module d'Young, pour une hygrométrie de $90 \%$ est comprise entre 3 et $5 \%$ d’un quartier récent à un vieux quartier alors que cette diminution n'est plus que 1 ou $3 \%$ pour les deux autres hygrométries et quel que soit l'âge considéré.

TABLEAUIII. Vitesses des déformations axiales et latérales pendant le fluage secondaire.

\begin{tabular}{c|c|c|c|c|c|c} 
& \multicolumn{2}{|c|}{ Quartier 2000 } & \multicolumn{2}{c|}{ Quartier 1970 } & \multicolumn{2}{c}{ Quartier 1920 } \\
\hline $\begin{array}{c}\text { Hygrométrie } \\
(\%)\end{array}$ & $\begin{array}{c}\text { Vitesse axiale } \\
\left(10^{-11} \cdot \mathrm{s}^{-1}\right)\end{array}$ & $\begin{array}{c}\text { Vitesse latérale } \\
\left(10^{-11}, \mathrm{~s}^{-1}\right)\end{array}$ & $\begin{array}{c}\text { Vitesse axiale } \\
\left(10^{-11} \cdot \mathrm{s}^{-1}\right)\end{array}$ & $\begin{array}{c}\text { Vitesse latérale } \\
\left(10^{-11} \cdot \mathrm{s}^{-1}\right)\end{array}$ & $\begin{array}{c}\text { Vitesse axiale } \\
\left(10^{-11}, \mathrm{~s}^{-1}\right)\end{array}$ & $\begin{array}{c}\text { Vitesse latérale } \\
\left(10^{-11} \cdot \mathrm{s}^{-1}\right)\end{array}$ \\
\hline 90 & 10,4 & 5,8 & 5,8 & 8,1 & 35,9 & 4,9 \\
60 & 0,3 & 0,9 & 4,6 & 2,3 & 2,3 & 4,8 \\
30 & 0,1 & $<0,1$ & 0.4 & 0,1 & 0,7 & $<0,1$ \\
\hline
\end{tabular}


TABLEAUIV Paramètres physiques déterminés avant et après les essais de fluage à hygrométrie contrôlée (le coefficient de Poisson dynamique avant et après essai est pris toujours égal à 0,33 ).

\begin{tabular}{|c|c|c|c|c|c|c|c|c|c|c|}
\hline Hygrométrie & $\begin{array}{l}\text { Paramètres } \\
\text { physiques }\end{array}$ & avant & $\begin{array}{l}\text { Quartien } \\
\text { après }\end{array}$ & $\begin{array}{l}2000 \\
\text { diminution } \\
\text { avant-après }\end{array}$ & avant & $\begin{array}{l}\text { Zuartier } \\
\text { après }\end{array}$ & $\begin{array}{l}1970 \\
\text { diminution } \\
\text { avant-après }\end{array}$ & avant & $\begin{array}{l}\text { Quartie! } \\
\text { après }\end{array}$ & $\begin{array}{l}1920 \\
\text { diminution } \\
\text { avant-après }\end{array}$ \\
\hline $90 \%$ & $\begin{array}{c}V_{p}(\mathrm{~m} / \mathrm{s}) \\
V^{p}(\mathrm{~m} / \mathrm{s}) \\
\mathrm{E}_{\mathrm{dym}}(\mathrm{GPa})\end{array}$ & $\begin{array}{l}4875 \\
2475 \\
37,5\end{array}$ & $\begin{array}{l}4850 \\
2425 \\
36,3\end{array}$ & $\begin{array}{l}<1 \% \\
<1 \% \\
3 \%\end{array}$ & $\begin{array}{c}5000 \\
2525 \\
38,6\end{array}$ & $\begin{array}{l}4975 \\
2450 \\
37,3\end{array}$ & $\begin{array}{l}<1 \% \\
3 \% \\
4 \%\end{array}$ & $\begin{array}{l}5025 \\
2525 \\
39,4\end{array}$ & $\begin{array}{l}4850 \\
2425 \\
36,4\end{array}$ & $\begin{array}{l}4 \% \\
4 \% \\
7 \%\end{array}$ \\
\hline $60 \%$ & $\begin{array}{l}V_{p}(\mathrm{~m} / \mathrm{s}) \\
V^{\prime}(\mathrm{m} / \mathrm{s}) \\
E_{d y m}(\mathrm{GPa})\end{array}$ & $\begin{array}{l}5000 \\
2525 \\
38,6\end{array}$ & $\begin{array}{l}5000 \\
2475 \\
37,9\end{array}$ & $\begin{array}{c}= \\
2 \% \\
2 \%\end{array}$ & $\begin{array}{l}4975 \\
2500 \\
38.5\end{array}$ & $\begin{array}{l}4875 \\
2450 \\
37.2\end{array}$ & $\begin{array}{l}2 \% \\
2 \% \\
3 \%\end{array}$ & $\begin{array}{l}5050 \\
2525 \\
39,0\end{array}$ & $\begin{array}{l}5000 \\
2500 \\
38,4\end{array}$ & $\begin{array}{c}1 \% \\
1 \% \\
<2 \%\end{array}$ \\
\hline $30 \%$ & $\begin{array}{l}V_{p}(\mathrm{~m} / \mathrm{s}) \\
V_{1}(\mathrm{~m} / \mathrm{s}) \\
E_{d i n}(\mathrm{GPa})\end{array}$ & $\begin{array}{l}5110 \\
2600 \\
42,1\end{array}$ & $\begin{array}{l}5040 \\
2550 \\
40,6\end{array}$ & $\begin{array}{l}<2 \% \\
2 \% \\
<4 \%\end{array}$ & $\begin{array}{l}5000 \\
2525 \\
38,9\end{array}$ & $\begin{array}{l}5000 \\
2500 \\
38,3\end{array}$ & $\begin{array}{c}= \\
1 \% \\
<2 \%\end{array}$ & $\begin{array}{l}5050 \\
2525 \\
39,2\end{array}$ & $\begin{array}{l}5000 \\
2525 \\
38,8\end{array}$ & $\begin{array}{l}1 \% \\
=1 \%\end{array}$ \\
\hline
\end{tabular}

\section{2}

\section{Observations microscopiques après essais de fluage}

Après les essais de fluage, des échantillons provenant de quartiers d'âge d'exploitation ancien (1920) ont été observés au MEB. Les observations sont systématiquement effectuées du bord vers le couur de l'échantillon sur un plan longitudinal et transversal. Ce plan d'observation passe par l'axe longitudinal de l'échantillon.

L'observation au MEB d'une éprouvette ayant subi un essai de fluage avec une hygrométrie de $30 \%$ nous a permis de relever différentes particularités:

- la taille des cristaux a diminué d'un facteur 10, elle devient inférieure à $25 \mu \mathrm{m}$ :

- il n'existe pas de frange de dissolution mais quelques traces de dissolution disséminées dans l'ensemble de l'éprouvette. Ces traces devaient être présentes avant l'essai de fluage.

Les observations sur une éprouvette ayant subi un essai de fluage avec une hygrométrie constante de $60 \%$ nous apportent les informations suivantes:

- la taille des cristaux a diminué d'un facteur 10, elle devient inférieure à $25 \mu \mathrm{m}$;

- une frange de dissolution concentrique, c'est-à-dire une frange où les cristaux sont émoussés, peut être observée sur une épaisseur de $1 \mathrm{~mm}$.

Vers le cour de l'éprouvette, quelques traces de dissolution d'une faible ampleur sont observées.

Pour une hygrométrie de $90 \%$, l'observation d'une éprouvette après l'essai de fluage a permis de relever différentes particularités:

- la taille des cristaux a diminué d'un facteur 10, elle devient inférieure à $25 \mu \mathrm{m}$;
- les éprouvettes présentent une importante densité de traces de dissolution: elles sont nettement plus nombreuses par rapport aux observations faites sur des échantillons témoins.

Malgré l'existence de traces de dissolution avant essai sur le gypse, on peut constater que l'hygrométrie entraîne une dissolution partielle de certains cristaux et que cette nouvelle dissolution varie dans le même sens que l'hygrométrie.

\section{3}

\section{Essais mécaniques après essais de fluage}

Afin d'essayer de vérifier l'existence d'un mécanisme d'endommagement par microfissuration du matériau pendant l'essai de fluage, des essais de compression isotrope ont été réalisés après les essais de fluage. Trois éprouvettes ont subi un essai de fluage sous une hygrométrie différente et les résultats des essais de compression isotrope (Tableau V) sont comparés avec ceux obtenus sur des échantillons témoins.

La porosité de fissure de référence mesurée sur les échantillons témoins est de $3.10^{-4}$. Seul l'échantillon ayant subi un essai de fluage avec une hygrométrie de $60 \%$ puis de $90 \%$ a une porosité de fissure deux fois supérieure à la porosité de référence. Le module de compressibilité des échantillons témoins diminue assez fortement suivant l'âge d'exploitation. On peut comparer également le module de compressibilité de l'échantillon "Quartier 2000 témoin » à celui de l'échantillon du quartier 2000 ayant subi les essais de fluage sous hygrométrie. Dans tous les cas le module de compressibilité des échantillons ayant subi des essais de fluage est inférieur. Ces constatations iraient dans le sens d'un endommagement de ces échantillons,

TABLEAU V Porosité de fissure et module de compressibilité calculés à partir des courbes contrainte-déformation des essais de compression isotrope.

\begin{tabular}{c|c|c|c|c|c} 
& Quartier 2000 témoin & Quartier 1920 térnoin & Quartier 1970 & Quartier 1970 \\
\hline Hygrométrie $(\%)$ & - & - & 30 & 60 \\
Porosité $\left(10^{-4}\right)$ & 3 & 3 & 4 & 3 \\
K $(\mathrm{MPa})$ & 38,3 & 30,4 & 33,4 & 60 puis 90 \\
\hline
\end{tabular}




\section{Interprétation-discussion}

L'interprétation-discussion des différents résultats peut se faire selon deux approches:

- pour un âge constant, le paramètre hygrométrie influe sur les résultats d'essais;

- pour une hygrométrie constante, le paramètre àge influe sur ces résuitats.

\section{1}

\section{Le paramètre hygrométrie}

Quel que soit l'âge d'exploitation considéré, la vitesse de fluage secondaire augmente avec l'hygrométrie. Pour un cas extrême, avec une hygrométrie de $90 \%$ et un âge d'exploitation ancien (1920), le fluage tertiaire a été atteint au bout de 45 jours.

Le changement d'hygrométrie, pour deux échantillons provenant du quartier en cours d'exploitation. permet de montrer que la vitesse de déformation de fluage est fonction de la valeur de l'hygrométrie. En effet, après ce changement d'hygrométrie, la vitesse s'accélère brutalement. Cependant, les vitesses de déformation du fluage secondaire n'ont pu être calculées car les essais ont été arrêtés avant d'obtenir une vitesse de fluage constante car les jauges d'extensométrie ne répondaient plus. Les vitesses des ondes ultrasonores $\mathrm{P}$ et $\mathrm{S}$ mesurées après l'essai de fluage ont diminué par rapport à celles qui ont été mesurées avant l'essai de fluage. La porosité de fïssure est deux fois plus importante par rapport à celle qui a été calculée sur des échantillons témoins.

Avec une hygrométrie de $90 \%$ et un ảge d'exploitation intermédiaire (1970), les vitesses des andes ultrasonores $\mathrm{P}$ et $\mathrm{S}$ mesurées après l'essai de fluage ont diminué par rapport à celles qui ont été mesurées avant l'essai de fluage. Suite à un essai de compression isotrope, la porosité de fissure reste inchangée par rapport à celle qui a été calculée sur des échantillons témoins. Les échantillons d'âge d'exploitation 1970 ayant subi un essai de fluage avec une hygrométrie de $60 \%$ et $30 \%$ présentent une porosité de fissure comparable à celle qui a été calculée sur des échantillons témoins.

Avec une hygrométrie de $90 \%$ et un áge d'exploitation ancien (1920), les vitesses des ondes ultrasonores P et S mesurées après l'essai de fluage ont diminué par rapport à celles qui ont été mesurées avant l'essai de fluage. Une observation au microscope électronique à balayage d'une éprouvette d'âge d'exploitation 1920 ayant subi un essai de fluage avec une hygrométrie de $60 \%$ a permis de mettre en évidence une frange de dissolution concentrique de $1 \mathrm{~mm}$ d'épaisseur et une diminution d'un facteur 10 de la taille des cristaux. L'éprouvette d'âge d'exploitation 1920 ayant subi un essai de fluage avec une hygrométrie de $30 \%$ présente quelques traces de dissolution qui devaient être présentes avant l'essai de fluage.

Les hypothèses permettant d'expliquer les variations des vitesses de fluage en fonction de l'hygrométrie sont basées sur des travaux antérieurs. D'après Finot et al. (1997), une faible hygrométrie entre 10 et $30 \%$ provoque une migration des molécules d'eau vers la périphérie des cristaux. En effet, une des deux molécules d'eau constitutive du gypse a une énergie de liaison plus faible que l'autre (Mandal et Mandal, 2002). Elle est alors considérée comme un anion. La conséquence de cette migration est la formation de dislocations dans les cristaux et la formation d'excroissances inférieures à 1 nanomètre. Proche de 100\% d'hygrométrie, la dissolution entraîne une formation de microfissures sur la face (010) et sur les extrémités de la face. Ces différentes observations ont été faites sur la surface d'un cristal de gypse naturel grâce à un microscope à force atomique.

Certaines traces de dissolutions observées au MEB existaient probablement avant les essaís de fluage et l'action de la vapeur d'eau a pu amplifier leur taille. Par conséquent, il n'est pas surprenant d'observer des pores mesurant quelques micromètres. Une sollicitation est appliquée en plus des conditions d'hygrométrie, le phénomène de dislocation ne peut ètre qu'amplifié provoquant le scindement d'un cristal sujvant les clivages secondaires dans les plans (100) et (011).

Une augmentation de la microfissuration a été mesurée uniquement sur une éprouvette ayant subi un essai de fluage avec un changement d'hygrométrie sans relâchement de la contrainte. La porosité de fissure après l'essai de fluage déterminée par un essai de compression isotrope était deux fois plus grande que celle des échantillons témoins. Cette variation peut être expliquée soit par une différence de la porosité initiale de cet échantillon, soit par une action combinée de la contrainte appliquée pendant l'essai de fluage et du changement de l'hygrométrie. La dispersion des mesures de la porosité de fissure sur les échantillons d'un même âge étant nettement plus faible que cette variation, notre choix va vers la deuxième hypothèse (action combinée de la contrainte appliquée pendant l'essai de fluage et du changement de l'hygrométrie). Les conséquences de l'hygrométrie sur la vitesse de fluage sont, semble-t-il, d'autant plus importantes que la roche est endommagée. En effet, Thygrométrie de $30 \%$, avec le niveau de contrainte des essais de fluage, n'induit pratiquement pas d'endommagement. A $60 \%$ puis $90 \%$ d'hygrométrie, l'endommagement devient sensible. Le changement d'hygrométrie sur cet échantillon a des conséquences à la fois sur les défauts injtiaux et sur ceux de l'endommagement, conduisant ainsi à une augmentation de la vitesse de fluage. II est donc clair, en terme d'hygrométrie, que le seuil de l'endommagement se situe quelque part entre $30 \%$ et $60 \%$. Nous ne connaissons pas actuellement comment ce seuil est également influence par l'état de contrainte. En effet, il est classiquement admis que l'endommagement dépend également de l'état de contrainte et, donc, plus giobalement, de la combinaison contrainte-hygrométrie.

Cette microfissuration mesurée uniquement sur une éprouvette ayant subi un essai de fluage avec un changement d'hygrométrie sans relâchement de la contrainte peut avoir une origine intergranulaire ou bien intragranulaire.

Pour le premier cas, les joints entre grains peuvent s'agrandir par une dissolution des surfaces cristallines. La frange de dissolution serait donc plus importante que celle obtenue pour l'échantillon maintenu dans une hygrométrie de $60 \%$. D'après les observations au MEB, tout l'échantillon a subi une attaque de la vapeur d'eau avec des figures de dissolution très marquées sur la 
périphérie et moins importantes vers le cœur. Ce type d'observation a déjà été décrit sur d'autres matériaux comme l'anhydrite (Dell'Angelo, 1995; Heilbronner, 1998) ou bien sur du quartz (Freeman, 1986) suite à des essais de fluage. Les forces de contact entre les surfaces cristallines parallèles à la direction [001] sont les plus faibles (Finot et al., 2001). Cette direction est celle qui a été observée au microscope électronique à balayage sur l'échantillon avec une hygrométrie maintenue à $30 \%$ : elle est proche de l'axe transversal de l'échantillon. C'est donc une direction privilégiée pour une augmentation de l'espace intergranulaire.

Le deuxième cas peut également être envisagé. En effet, un débit lamellaire plus important que celui qui a été observé sur un échantillon maintenu à $60 \%$ d'hygrométrie est tout à fait envisageable. D'après Finot et al. (1997), une hygrométrie proche du pôle $100 \%$ engendre une microfissuration sur la surface cristalline $(010)$ et sur ces extrémités (Sipple et al., 2001) dans la direction [100]. Cette microfissuration peut entrainer le scindement d'un cristal en plusieurs autres cristaux plus petits. L'augmentation des traces de dissolution n'est pas étonnante car d'après plusieurs auteurs (Christoffersen, 1976; Amathieu, 1988; Raines, 1997; Jeschke, 2001), la vitesse de dissolution dans une eau saturée à $50 \%$ de gypse est de $10^{-5} \mathrm{mmol} \cdot \mathrm{cm}^{-2} \cdot \mathrm{s}^{-1}$.

Pour conclure, la dissolution des minéraux observée sur le gypse après les essais de fluage est due à l'action de la vapeur d'eau sous contrainte (mécanisme de pression-dissolution sans précipitation observable).

\section{2}

\section{Le paramètre âge de l'exploitation}

Quelle que soit I'hygrométrie considérée, la vitesse de fluage secondaire augmente avec l'ancienneté de l'exploitation. Cette augmentation est beaucoup plus marquée pour une hygrométrie de $90 \%$.

La vitesse de déformation axiale pendant le fluage secondaire a augmenté de $30 \%$ entre un échantillon provenant d'un quartier récent et un échantillon d'âge d'exploitation ancien. La vitesse de déformation latérale pendant cette même phase a augmenté de $13 \%$. Ces vitesses ont été calculées lors d'un essai de fluage avec une hygrométrie égale à $90 \%$. Avec une hygrométrie constante de $60 \%$, les vitesses des déformations axiales et latérales pendant la phase de fluage secondaire ont augmenté respectivement de $15 \%$ et de $20 \%$ entre un échantillon provenant d'un quartier récent et un échantillon d'âge d'exploitation ancien. Enfin, pour une hygrométrie constante de $30 \%$, l'augmentation de la vitesse de déformation axiale de la phase de fluage secondaire est de $15 \%$ entre un échantillon provenant d'un quartier récent et un échantillon d'àge d'exploitation ancien. Cependant, la vitesse de déformation latérale est restée constante.
Les hypothèses permettant d'expliquer les variations de vitesses de fluage en fonction de l'âge d'exploitation sont les suivantes:

- peu de différences texturales et structurales peuvent expliquer totalement les variations de vitesse de fluage. En effet, seules quelques torsions de cristaux avec un débit lamellaire et une diminution de la taille des cristaux ont été observées sur les échantillons des quartiers anciens. Cependant, ces différences cristallines peuvent participer à ces variations de vitesse de fluage. Après les essais de fluage, les différences sont beaucoup plus marquées avec une forte diminution de la taille des cristaux. Pour les hygrométries de $60 \%$ et $90 \%$, des figures de dissolution importantes apparaissent en grand nombre. Dans ce cas, le rôle de l'hygrométrie devient un paramètre majeur;

- la différence dans les paramètres mécaniques définis lors de la caractérisation mécanique court terme du gypse provenant de quartiers d'âge d'exploitation différent (Auvray, 2003) pourraient expliquer en partie les variations de vitesse de fluage. En effet, une diminution de la résistance en compression simple existe entre des échantillons partiellement saturés provenant d'un quartier d'âge d'exploitation récent et d'un quartier d'âge d'exploitation ancien. Cette diminution de résistance est d'autant plus importante que l'hygrométrie est élevée.

Il semble que les différences texturales et structurales et les faibles différences des paramètres mécaniques entre les échantillons d'âge d'exploitation différent, avant les essais de fluage, aient une importance sur les variations de vitesse de fluage pendant les essais de fluage.

7

\section{Conclusion}

Le gypse de Grozon présente des traces de dissolution de plus en plus marquées avec l'âge d'exploitation. L'intensité de la dissolution décroit avec la profondeur, du parement vers le cœur du pilier. Ces faits suggèrent un vieillissement de la roche fonction du temps. La dégradation des piliers est donc probablement due au vieillissement du massif rocheux associé au comportement différé du matériau. Ce comportement différé est fortement influencé par l'hygrométrie et par l'âge d'exploitation des quartiers. L'action de l'humidité de l'air sur les cristaux de gypse des piliers ou bien des échantillons ayant subi un essai de fluage à hygrométrie contrôlée entraîne leur dégradation. Elle se manjfeste par une c corrosion $x$ des surfaces cristallines. Le comportement différé est influencé par le couplage hydro-chimico-mécanique. Les vitesses de fluage secondaire les plus élevées sont mesurées sous une forte hygrométrie. De plus le comportement mécanique différé est fonction de l'âge d'exploitation du gypse et donc de son vieillissement. 
Amathieu L., Boistelle R. - Crystallization kinetics of gypsum from dense suspension of hemihydrate in water. Journal of Crystal Growth, vol. 88, Issue 2, 1988, p. 183-192.

Auger F. - Viellissement par altération atmosphérique des matériaux de construction - Étude comparative in situ et en simulation. Colloque intern. Détérioration des matériaux de construction. La Rochelle, 1991, p. 115-129.

Auvray C. - Vieillissement et comportement rhéologique, Thèse de doctorat INPL, décembre 2003, 268 p.

Chéne G. Bastian G. Bruniail C. Laurent J.P. - Vleillissement accéléré de bloc de tuffeau en laboratoire sous l'effet de cycles d'imbibition-séchage. Matériaux et constructions, vol. 32, 1999, p. 525-532.

Christoffersen J.. Christoffersen M.R. - The kinetics of dissolution of calcium sulphate dihydrate in water. Journal of Crystal Growth, vol. 35. Issue 1, 1976, p. $79-88$.

Dell'Angello L.N., Olqaard D.L. - Experi mental deformation of fine-grained anhydrite: evidence for dislocation and diffusion creep. J. of Geophysical Research, vol. 100, 1995, p. 15425-15440.

Farran J., Thenoz B. - L'altérabilité des roches, ses facteurs, sa prévision. Suppl. Annales de ITTBTP n० 215, 1965, 27292736.

Finot E., Lesniewska E., Goudonnet J.P., Mutin J.C., Domenech M., Kadi A.A. (2001). Correlating surface forces with surface reactivity of gypsum crystals by atomic force microscopy. Comparaison with rheological properties of plaster. Solid State Ionics, vol. 141-142, 2001, p. 39-46.

Finot E., Lesniewska E., Mutin J.C., Goudonnet J.P. - Reactivity of gypsum faces according to the relative humidity by scanning force microscopy. Surface Science, vol. 384, 1997, 201-217.
Freeman B., Ferguson C.C.-Deformation mechanism and micromechanism of rocks with distributer grain sizes, $J$. of Geophysical Research, vol. 91, 1986 p. 3849-3860.

Furlan V., Girardet F, - Pollution atmosphérique et durabilité des pierres de construction. Colloque intern. Détérioration des matériaux de construction, La Rochelle, 1991, p. 79-91.

Grgic D., Homand F., Dagallier G. - Altération du minerai de fer dans les mines abandonnees de Lorraine. Revue francrise de géotechnique, $\mathrm{n}^{\circ}$ 99, 2002, p. 49-60.

Griggs D.T. - Creep of rocks. Journal of Geology, vol. 47, 1939, p. 225-251.

Griggs. D.T. - Experimental flow of rocks under conditions favouring recrystallisation. Geol. Soc. Amer. Bull., vol. 51, 1940, p. 1001-1022.

Griggs D.T. Handin - Rocks deformation. Geol. Soc. Amer. Mem. vol. 79, 1960.

Gupta A.S., Rao K.S. - Weathering effects on the strength and deformational behaviour of crystalline rocks under uniaxial compression state. Engineering Geology, vol. 56, 2000, p. 257-274.

Heiinen W.M.M. Hartman P. - Structural morphology of gypsum $\left(\mathrm{CaSO}_{4} 2 \mathrm{H}_{2} \mathrm{O}\right)$, brushite (CaHPO $2 \mathrm{H}_{2} \mathrm{O}$ ) and pharmacolite (CaHAsO, 2H, O). Journal of Crystal Growth, vol. 108, 1991, p. 290-300.

Heilbronner R., Bruhn D. - The influence of three-dimensional grain size distributions on the rheology of polyphase rocks, J. of Structural Geology, vol. 20, $n^{\circ}$ 6, 1998, p. 695-705.

Jeschke A.A., Vosbeck K., Dreybrodit WSurface controlled dissolution rates of grpsum in aqueous solutions exhibit nonlinear dissolution kinetics. Geochimica et Cosmechimica Acta, vol. $65, n^{\circ} 1$. 2001, p. $27-34$
Jong W.F., Bouman J. - General crystallography, a brief compendium. Freeman and Co. Ed., 1959, 281 p.

Mandal P.K.. Mandal T.K. - Anion water in gypsum $\left(\mathrm{CaSO}_{4}, 2 \mathrm{H}_{2} \mathrm{O}\right)$ and hemihydrate (CaSO $1 / 2 \mathrm{H}, \mathrm{O})$. Cement and Concrete Research, vol. 32, 2002, p. 313-316.

Morlier P.J. - Étude expérimentale de la déformation des roches. Thèse Université de Paris, Faculté des sciences, 1964. $40 \mathrm{p}$.

NF EN ISO 483 - Petites enceintes de conditionnement et d'essai utilisant des solutions acqueuses pour maintenir I'humidité relative à une valeur constante. Afnor, 1999.

NF X 15-119-Mesure de l'humidité de l'air, Générateurs d'air humide à solutions salines pour l'étalonnage des hygromètres. Afnor, 1999.

Ogniben L. Inverse graded bedding in primary gypsum of chemical deposition. Journal of Sedimentary Petrology, vol. 25, 1955, p. 273-281.

Raines M.A., Dewers T.A. - Mixed transport/reaction control of gypsum dissolution kinetics in aqueous solution and initiation of gypsum karst. Chemical Geology, vol. 40, 1997, p. 29-48.

Sipple E.M. - Réarrangements structuraux et modifications microstructurales associés aux transformations de phases dans le système CaSO $(\mathrm{s}) \mathrm{H}_{\mathrm{H}} \mathrm{O}(\mathrm{g})$, Thèse de doctorat de l'université de Bourgogne, $1999,140 \mathrm{p}$.

Sipple E.M. Braconni P. Dufour P. Mutin J.P. - Microstructural modifications resulting from the dehydration of gypsum. Solid State lonics, vol. 141-142. 2001, p. 447-454.

Williams S.C. - The shear strength of gypsum single crystal on three cleavage planes. Tectonophysics, vol. 148, 1988, p. 163-173. 